\title{
Single-Trial EEG Classification Using Logistic Regression Based on Ensemble Synchronization
}

\author{
Pradeep D. Prasad, Member, IEEE, Harsha N. Halahalli, John P. John, \\ and Kaushik K. Majumdar, Senior Member, IEEE
}

\begin{abstract}
In this paper, we propose an ensemble synchronization measure across all EEG channel pairs of a cluster based on Frobenius norm of the phase synchronization matrix, in a 0-1 scale enabling a direct comparison between clusters with different number of channels. Using this metric, we studied the intrahemispheric EEG synchronization in the lower gamma band $(30-40 \mathrm{~Hz})$ during 1229 single trials of an audio-visual integration cross modal task (CMT) recorded from five patients with schizophrenia and five healthy control subjects. Using ensemble synchronization measure and response latency of single trials recorded during the CMT as features for logistic regression, we could classify each single trial of EEG as belonging to a patient with schizophrenia or a healthy control subject with $73 \%$ accuracy, with an area under receiver operating characteristics curve of 0.83 . We also propose a likelihood rating to denote the possibility of a subject belonging to the schizophrenia group.
\end{abstract}

Index Terms-Cross modal task (CMT), Frobenius norm, Hilbert transform, logistic regression, multichannel measure, phase synchronization.

\section{INTRODUCTION}

$\mathbf{H}$ UMAN cognitive functions involve the functional integration among different brain regions that are specialized for certain aspects of cognitive processing [1]. To perform even a simple task, the brain simultaneously employs multiple functionally distinct areas. Phase synchrony of neuronal oscillations has been postulated to be an important mechanism for binding together the activity of different brain regions in order to generate a unified perceptual experience from multiple sensory stimuli. This has been referred to as the binding problem by Treisman [2]. Phase synchronization has been implicated in sensory perception [3], attention modulation [4], memory processes [5],

Manuscript received December 30, 2012; revised June 20, 2013, August 4 2013 and September 23, 2013; accepted October 24, 2013. Date of publication December 12, 2013; date of current version May 1, 2014. This work was supported in part by an internal research grant of the Indian Statistical Institute and the Department of Biotechnology, Government of India under Grant BT/PR/8363/14/1252.

P. D. Prasad and K. K. Majumdar are with the Systems Science and Informatics Unit, Indian Statistical Institute, Bangalore 560059, Karnataka, India (e-mail: pradeepd@ieee.org; mkkaushik@hotmail.com).

H. N. Halahalli is with the Department of Physiology, K. S. Hegde Medical Academy, Nitte University, Mangalore 575018, India (e-mail: hnharsha@ gmail.com).

J. P. John is with the Multimodal Brain Image Analysis Laboratory, Department of Psychiatry and Department of Clinical Neuroscience, National Institute of Mental Health and Neurosciences, Bangalore 560029, Karnataka, India (e-mail: jpjnimhans@gmail.com).

Color versions of one or more of the figures in this paper are available online at http://ieeexplore.ieee.org.

Digital Object Identifier 10.1109/JBHI.2013.2289741 neural communication [6], synaptic plasticity [7], action planning [8], task execution [9] etc. Schizophrenia, a neuropsychiatric condition characterized by aberrant brain connectivity [1], has been shown to be associated with task specific abnormal synchrony in scalp EEG channels [10]. Therefore, we examined scalp EEG phase synchronization across different brain regions during the performance of an audio-visual integration task, in the hope of differentiating subjects with schizophrenia from healthy controls using a novel ensemble synchronization-based classification metric.

Task specific single-trial EEG classification, based on signatures of brain activities, has remained a challenging problem [11]-[13]. In the past, differentiation of patients with schizophrenia from healthy controls has been attempted using artificial neural networks on EEG with $71 \%$ accuracy [14]. In this study, our focus was on single-trial EEG classification between schizophrenia and healthy groups. The need to use single trials came from our intent of developing a real-time system capable of performing such a classification. Here, we studied ensemble phase synchronization as a feature for classification, where the ensemble would encompass all brain regions involved in performing those cognitive tasks where a difference in performance between subject groups is expected. We chose to study intrahemispheric ensemble synchronization in lower gamma band, which is involved in long-range coordination [15], as there are reports suggesting dysfunctional long-range coordination in schizophrenia [16], [17].

In the next section, we describe the experiment and data acquisition. In Section III, we present a theoretical description of the quantitative methods used to process the data. In Section IV, we present the results. The paper concludes with a Conclusion section.

\section{EXPERIMENT AND DATA}

\section{A. Subjects}

Scalp EEG of five right-handed patients with schizophrenia and five healthy right-handed healthy control subjects were randomly selected from an EEG data base of schizophrenia and healthy subjects acquired as part of a funded research project at the National Institute of Mental Health and Neurosciences (NIMHANS), Bangalore, India. The earlier study had the approval of the NIMHANS Ethics Committee, thus conforming to the ethical standard laid down in the 1964 Declaration of Helsinki. Written informed consent was obtained from all participants, prior to enrolling them into the study. The sociodemographic details of all the subjects are given in 
TABLE I

DEMOGRAPHIC AND Clinical CHARACTERISTICS OF PATIENTS WITH SCHIZOPHRENIA AND HEALTHY CONTROLS (UPPER PANEL) ALONG With BETWEEN-GRoup COMPARISONS ON THE ABOVE VARIABLES (LOWER PANEL)

\begin{tabular}{|c|c|c|c|c|}
\hline \multirow{2}{*}{ Variable } & \multicolumn{2}{|c|}{$\begin{array}{c}\text { Patients with } \\
\text { Schizophrenia (SZ) }\end{array}$} & \multicolumn{2}{c|}{$\begin{array}{c}\text { Healthy Control } \\
\text { subjects (HS) }\end{array}$} \\
\cline { 2 - 5 } & Mean & CI & Mean & CI \\
\hline Age (years) & 31.8 & \pm 12.1 & 23.8 & \pm 1.8 \\
\hline Sex (male/female) & $4 / 1$ & & $3 / 2$ & \\
\hline Education (years) & 10.4 & \pm 6.9 & 15.6 & \pm 1.9 \\
\hline $\begin{array}{c}\text { Duration of } \\
\text { Illness (months) }\end{array}$ & 37.8 & & - & - \\
\hline
\end{tabular}

\begin{tabular}{|c|c|c|c|c|}
\hline \multirow{2}{*}{ Variable } & \multicolumn{2}{|c|}{ Significance level } & \multicolumn{2}{c|}{$\begin{array}{c}\text { Chi squared test } \\
\text { for independence }\end{array}$} \\
\cline { 2 - 5 } & $\begin{array}{c}\mathrm{t}_{(\mathrm{df})} \\
\text { Statistic }\end{array}$ & $\begin{array}{c}\mathrm{p}- \\
\text { value }\end{array}$ & $\begin{array}{c}\chi^{2}{ }_{(\mathrm{df})} \\
\text { Statistic }\end{array}$ & $\begin{array}{c}\mathrm{p}- \\
\text { value }\end{array}$ \\
\hline Age (years) & $\mathrm{t}_{(8)}=1.8$ & 0.1 & $\chi_{(8)}^{2}=10$ & 0.26 \\
\hline Sex (male/female) & & & $\chi_{(1)}^{2}=0$ & 1 \\
\hline Education (years) & $\mathrm{t}_{(8)}=-2$ & 0.08 & $\chi_{(6)}^{2}=7$ & 0.32 \\
\hline $\begin{array}{c}\text { Duration of } \\
\text { Illness (months) }\end{array}$ & & & $\chi_{(4)}^{2}=5$ & 0.29 \\
\hline
\end{tabular}

TABLE II

TREATMENT Status AND CliniCAL CHARACTERISTICS OF PATIENTS WITH SCHIZOPHRENIA

\begin{tabular}{|c|c|c|c|}
\hline $\begin{array}{c}\text { Schizophrenia } \\
\text { Patient } \\
\text { Identifier }\end{array}$ & $\begin{array}{c}\text { Age of } \\
\text { onset of } \\
\text { illness } \\
\text { (years) }\end{array}$ & $\begin{array}{c}\text { Illness } \\
\text { duration } \\
\text { (months) }\end{array}$ & $\begin{array}{c}\text { Treatment status } \\
\text { (see legend) }\end{array}$ \\
\hline SZ003 & 39 & 6 & NN \\
\hline SZ007 & 30 & 120 & MF \\
\hline SZ010 & 24 & 36 & RT \\
\hline SZ012 & 17 & 3 & NN \\
\hline SZ013 & 34 & 24 & MF \\
\hline
\end{tabular}

NN- Neuroleptic-naïve; MF- medication-free for 2 months or more; RT- on regular treatment.

TABLE III

Total Number of TRIALS SELECTED For EACH CATEGORY AND STIMULUS TYPE

\begin{tabular}{|c|c|c|c|}
\hline $\begin{array}{c}\text { Stimulus } \\
\text { type }\end{array}$ & $\begin{array}{c}\text { Total } \\
\text { selected } \\
\text { trials }\end{array}$ & $\begin{array}{c}\text { Healthy } \\
\text { control } \\
\text { subjects }\end{array}$ & $\begin{array}{c}\text { Schizophrenia } \\
\text { subjects }\end{array}$ \\
\hline Congruent & 376 & 200 & 176 \\
\hline Incongruent & 360 & 190 & 170 \\
\hline Neutral & 493 & 259 & 234 \\
\hline
\end{tabular}

Table I, while the clinical details are listed in Table II. Statistically significant difference between groups for each variable was evaluated by conducting independent two sample $t$-test. Results of Pearson's chi-squared test for independence have been reported for sake of completeness. A larger sample is required to draw conclusions from these metrics confidently.

\section{B. Equipment Configuration}

The EEG was acquired using a 70-channel Neuroscan SynAmps acquisition system (Compumedics, Charlotte, USA). An electrode cap with the 10-10 International system of electrode placement was used. This included 64 monopolar scalp electrodes and four bipolar electrodes for EOG, EKG, and EMG. Acquisition was controlled using ACQUIRE, Neuroscan's proprietary acquisition software. Impedances for all electrodes were reduced to a range of 5-10 $\mathrm{K} \Omega$. A high-pass filter of $0.1 \mathrm{~Hz}$ and a low-pass filter of $100 \mathrm{~Hz}$ were used at the time of recording. Sample frequency was set to $1000 \mathrm{~Hz}$. Stimulus delivery was controlled by STIM2 (Compumedics, Charlotte, USA), a stimulus presentation system which includes a dedicated audio system and a four-button response pad for collection of fingerpress responses from the subjects. The subjects were seated in a comfortable cushioned chair in a darkened, sound-attenuated room facing a $17^{\prime \prime}$ CRT monitor at eye-level which was connected to the STIM2 system.

\section{Cross Modal Task (CMT)}

This is an audio-visual integration task administered as an event-related paradigm and designed to engage multimodal perceptual integration processes in the brain. The subjects were simultaneously presented with an image on a computer monitor and a sound through earphones. The gestalt image could be a human male or female face or a scrambled image. The sound could be a snippet of a human male or female voice or white noise. The subject's task was to pay close attention to both the visual and auditory stimuli and to respond by appropriate button press if the gender of the face in the image and the voice in the sound matched (congruent trials, index finger press), or did not match (incongruent trials, middle finger press) or if there was no discernible face or voice (neutral trials, ring finger press). Trials in which no image was presented, served as null trials to provide stimulus free baseline while also ensuring that the intertrial interval is random.

Of a total of 256 trials for each subject, $25 \%$ each were congruent, incongruent, neutral, and null trials. The sequence of image-voice combination was prespecified according to a pseudorandom list such that the appearances of each stimulus type were approximately equal during any interval. Half of the incongruent trials had male face-female voice pairings and the other half had female face-male voice pairings. For the neutral task, the scrambled image and white noise clipping were paired.

At the onset of each trial, a fixation cross-hair was presented which lasted for $2060 \mathrm{~ms}$ before being replaced by the probe image. Stimulus onset for both visual and auditory stimuli was $2060 \mathrm{~ms}$ after trial onset. The size of visual stimuli was $180 \times 200 \mathrm{px}$ which subtended a visual angle of $6.9^{\circ}$ vertically and $6.2^{\circ}$ horizontally at a viewing distance of $60 \mathrm{~cm}$. The duration of both visual and auditory stimuli was $500 \mathrm{~ms}$. The fixation cross-hair reappeared $1500 \mathrm{~ms}$ after the stimulus offset, with intertrial interval being $5070 \mathrm{~ms}$. Fig. 1 captures the sequence of events for each trial in the CMT EEG experiment.

The data on acquisition was band pass filtered between 0.5 and $80 \mathrm{~Hz}$ and notch filtered at $49-51 \mathrm{~Hz}$ to remove the acline noise. ECG and EMG channels were removed. Artifacts were rejected upon visual inspection and by ICA-based methods using ADJUST toolbox [18], [19] in EEGLAB [20] a MATLAB toolbox for processing EEG. 


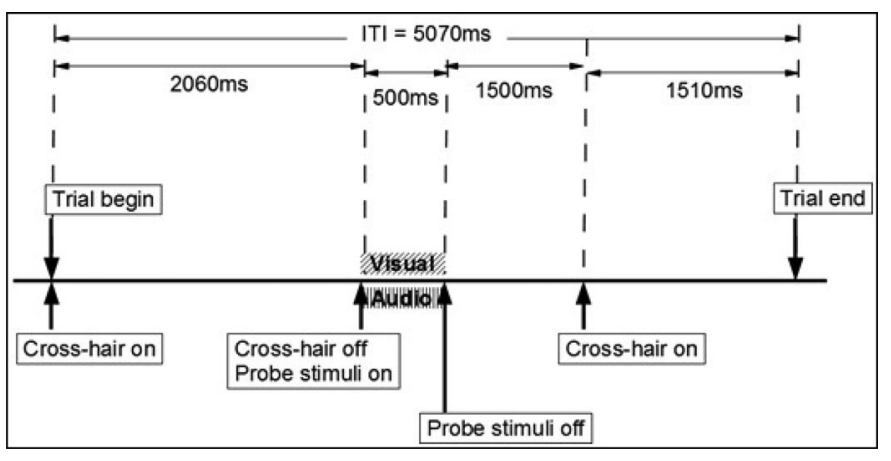

Fig. 1. Diagram illustrating the timeline for each trial in the CMT EEG experiment.

\section{QuANTITATIVE Methods}

\section{A. Hilbert Phase Synchronization}

Let $s(t)$ be any time domain signal. Let us define analytic signal $\psi(t)$

$$
\psi(t)=s(t)+j \cdot \hat{s}(t)=A(t) \cdot \exp (j \cdot \varphi(t))
$$

where $\hat{s}(t)$ is the Hilbert transform of the signal $s(t), j=\sqrt{-1}, A(t)$ is the instantaneous amplitude (envelope), and $\varphi(t)$ is the instantaneous phase of the analytic signal $\psi(t)$. For phase to be free from influence of amplitude, the signal must be narrow band [21]. So we consider the lower gamma (30-40 Hz) band for the phase synchronization analysis, which is sufficiently narrow and also includes the $40-\mathrm{Hz}$ representative component instead of entire gamma band [22] instead of the entire 30-80 Hz [23]-[26]. Instantaneous phase difference $\varphi(t)$ at time $t$ is $\phi_{12}(t)=\phi_{1}(t)-\phi_{2}(t)$ where $\varphi_{1}, \varphi_{2}$ phases of first and second EEG channels of the pair are. Finally, we define the synchronization value $s$ between a channel pair for window $W_{t}$ as follows:

$$
s_{12}\left(W_{t}\right)=\left|\frac{1}{|W|} \sum_{t=1}^{|W|} \exp \left(i \cdot \phi_{12}(t)\right)\right|^{2}
$$

where $|W|$ is the length of the analysis window $W$ [27]. If $s=1$ signal pairs are in perfect phase synchrony and if $s=0$ the signals are in perfect phase asynchrony. If there are $n$ number of EEG channels in a cluster an $n \times n$ symmetric matrix $A_{n}(t)$ is created whose $i j$ th entry $a_{i j}(t)$ is the phase synchronization of $i$ th and $j$ th channels as calculated in (2).

The EEG signals acquired during the CMT were analyzed by segmenting into trials from $1 \mathrm{~s}$ before the stimulus onset till 2 afterward. The data were band pass filtered for lower gamma band using an equiripple FIR filter with the following specification: pass bands at 30 and $40 \mathrm{~Hz}$; stop bands at 26 and $44 \mathrm{~Hz}$; pass-band and stop-band attenuation of 0.5 and $40 \mathrm{~dB}$, respectively. The analysis window length used for calculating phase synchronization was $400 \mathrm{~ms}$, which was shifted by one time point at a time. We obtained two $35 \times 35$ symmetric matrices one for each hemisphere, as all eight channels on the center line are included in both hemispheres i.e., $((62-8) / 2)+8=35$ channels in each hemisphere.

\section{B. Ensemble Synchronization Measure}

We propose a quantitative measure to define an ordering of the synchronization matrices based on Frobenius norm of a matrix [28]. If $a_{i j}$ is the $i j$ th entry of a matrix $A$, then the entry wise norm $\|A\|_{p}$ is defined in (3) where $p$ is a positive integer and $n$ is the number of channels in the ensemble. We chose Frobenius norm $\|A\|_{F}$, by substituting $p=2$ as follows:

$$
\begin{aligned}
\|A\|_{p} & =\left(\sum_{i=1}^{n} \sum_{j=1}^{n}\left(a_{i j}\right)^{p}\right)^{1 / p} \\
\gamma & =\sqrt{\frac{\|A\|_{F}^{2}-n}{n^{2}-n}} .
\end{aligned}
$$

The ensemble measure is computed on a $0-1$ scale as given in (4), where $n$ is the number of channels in cluster. Here, a value of 0 corresponds to the lowest ensemble synchronization while 1 corresponds to highest. This enables comparison of different clusters using the proposed ensemble measure, thus one can tell which ensemble or which subject exhibits greater synchronization.

\section{Statistical Significance of Phase Synchronization}

We test the null hypothesis $H_{0}$, that the observed phase synchronization on a channel pair $s_{1,2}$ is not statistically significant or $H_{0}: s_{1,2}=0$. The decision rule used to test this hypothesis is: reject $H_{0}$ if $s_{1,2}>s_{0}$, where for a given $p$-value, $s_{0}$ is the $100 \times(1-p)$ percentile of the distribution of $s$ values obtained as phase synchronization between a large number of pairs of independent shifted-time surrogate signals [29]. For Hilbert transform-based pairwise phase synchronization over an analysis window of $400 \mathrm{~ms}$, we observe $s_{0}=0.51$ when evaluated over 100 pairs of surrogate signals for a $p$-value of 0.05 or $95 \%$ significance level. We observe a high value for $s_{0}$ since the analysis window duration is small. The choice of window length is influenced by our desire to retain good temporal resolution to facilitate in capturing the changing dynamics.

Now, we conduct a similar null hypothesis significance testing on the proposed ensemble synchronization measure $\gamma$. The decision rule used to test null hypothesis $H_{0}$, i.e., observed ensemble synchronization is not significant is to reject $H_{0}$ if $\gamma>\gamma_{0}$, where $\gamma_{0}$ is evaluated as the $100 \times(1-p)$ percentile of the distribution of $\gamma$ values evaluating on a large number of ensembles of independent shifted-time surrogate signals for a given $p$-value. Using the proposed ensemble synchronization measure $\gamma$, we observe $\gamma_{0}=0.51\left(=s_{0}\right)$ for a 100 surrogate ensemble with 35 channels each for a $p$-value of 0.05 . Even intuitively there is no reason to expect ensemble synchronization to be significant when pairwise synchronization is not or vice versa. As expected the statistical significance threshold for ensemble synchronization $\gamma_{0}$ is similar to the synchronization threshold for channel pair $s_{0}$ irrespective of the number of signals in the ensemble. 


\section{Logistic Regression}

Let $m, n$ be number of training examples, and features extracted from data. Transpose is denoted as $(\bullet)^{T}$. The feature matrix $X$ is defined as follows:

$$
\begin{aligned}
& X=\left[\left(x^{(1)}\right)^{\mathrm{T}}\left(x^{(2)}\right)^{\mathrm{T}} \cdots\left(x^{(i)}\right)^{\mathrm{T}} \cdots\left(x^{(m)}\right)^{\mathrm{T}}\right]^{\mathrm{T}}
\end{aligned}
$$

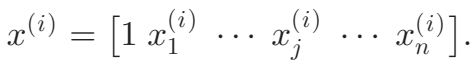

Here $x^{(i)}$, of order $1 \times(n+1)$, is the feature vector of $i$ th example and $x_{j}^{(i)}$ is its $j$ th feature. The extra dimension corresponds to the intercept term set to 1 , i.e., $x_{0}^{(i)}=1$. Now, $i$ th example is represented as $\left(x^{(i)}, y^{(i)}\right)$, where $y^{(i)}$ in (6) defines target class vector $Y$ of order $m \times 1$ :

$$
y^{(i)}= \begin{cases}0, & \text { healthy control } \\ 1, & \text { schizophrenia. }\end{cases}
$$

The hypothesis function $h$ is the sigmoid which restricts the output to $[0,1]$ range. The output $h_{\theta}\left(x^{(i)}\right)$ corresponding to $\left(x^{(i)}, y^{(i)}\right)$ defined in (7), depends on a weight vector $\theta$ of length $1 \times(n+1)$ :

$$
h_{\theta}\left(x^{(i)}\right)=\frac{1}{\left(1+\exp \left(-x^{(i)} \cdot \theta^{\mathrm{T}}\right)\right)} .
$$

The output $h_{\theta}\left(x^{(i)}\right)$ is the score assigned to each single trial ( $i$ th example) and its predicted class is decided using (8) single trial $\left(x^{i}, y^{i}\right)$ is given by

$$
\left(x^{(i)}, y^{(i)}\right)= \begin{cases}\text { healthy control, } & \text { if } h_{\theta}\left(x^{(i)}\right)<0.5 \\ \text { schizophrenia, } & \text { if } h_{\theta}\left(x^{(i)}\right) \geq 0.5 .\end{cases}
$$

The goal is to minimize a cost function by searching for a weight vector $\theta_{\min }$ such that a hyper plane partitions the examples into its respective classes with best possible accuracy. To avoid overfitting for training dataset leading to a loss of generalization, we perform regularization, penalizing the cost function with weights $\theta$ and degree of regularization is controlled by parameter $\lambda$.

\section{E. Logistic Regression-Based Classification}

We chose four features for the classifier viz. average hemispheric synchronization measures evaluated using the proposed ensemble measure (4) over a $300 \mathrm{~ms}$ window at three intervalsat stimulus onset (interval from stimulus onset to $300 \mathrm{~ms}$ afterward), at stimulus offset (interval from stimulus offset to $300 \mathrm{~ms}$ afterward) and at response time (interval from 400 to $100 \mathrm{~ms}$ preresponse) apart from using response latency.

We restricted the trials that are used for the classification by the following criteria. Apart from rejecting trials at preprocessing stage, due to spurious signals, noise, etc., we chose only trials with a correct response. Further, we selected trials in which the response latency is at most two standard deviations away from the mean response latency for the subject under consideration. This ensures that trials where the subject responded either very quickly or delayed are not considered as they might have been a random guess due to haste or confusion. Summary of number of trials selected for each category and stimulus type is presented in Table III.

\section{F. Classifier Performance}

For each stimulus type, we trained a logistic regression classifier on randomly chosen subset of the selected trials that were divided into training set $(65 \%)$ and testing set $(35 \%)$ using a pseudorandom sequence. Then we compute the performance on testing dataset by using $\theta_{\text {min }}$ obtained on the training set with regularization parameter set to 1 . The performance of the classifier is evaluated using accuracy at single trial on training set in tandem with testing set which reflects the real-world scenario. Further, we report sensitivity, i.e., true-positive rate defined as ratio of true positives to actual number of positive in the total dataset using a threshold of 0.5 , and false-positive rate defined as the ratio of false positives to the actual number of negatives in the dataset using a threshold of 0.5 , this is equivalent to (1- specificity).

\section{G. Likelihood Rating}

A likelihood rating lik $\left(s_{j}\right)$ is assigned to each subject $s_{j}$, which reflects the likelihood of the subject as belonging to schizophrenia category over healthy control. This rating ranges from 0 denoting healthy, to 1 denoting schizophrenia, computed as the average of scores assigned to all selected single trials $\operatorname{tr}\left(s_{j}\right)$ belonging to subject $s_{j}$ for a particular stimulus type, where score $\left(i, s_{j}\right)$ is the score assigned to $i$ th selected single trial defined as the predicted output of the classifier $h_{\theta_{\min }}\left(x^{(i)}\right)$ (see (7)). If the likelihood rating is greater than threshold then the subject is termed as belonging to schizophrenia category, else to healthy category. In our study, a threshold of 0.5 is chosen for performing the subject level classification:

$$
\begin{gathered}
\operatorname{lik}\left(s_{j}\right)=\frac{1}{\operatorname{tr}\left(s_{j}\right)} \sum_{i=1}^{\operatorname{tr}\left(s_{j}\right)} \operatorname{score}\left(i, s_{j}\right) \\
s_{j} \in \begin{cases}\text { healthy control, } & \text { lik }\left(s_{j}\right)<\text { threshold } \\
\text { schizophrenia, } & \text { lik }\left(s_{j}\right) \geq \text { threshold. }\end{cases}
\end{gathered}
$$

\section{RESULTS}

\section{A. Behavioral Results}

The behavioral performance during the CMT trials is given in Table IV. The mean and 95\% confidence interval for response accuracy and response latency for both groups across all the three stimulus types are listed. The mean difference in response latency between groups was statistically significant for congruent and incongruent but not for neutral stimulus type. Therefore, response latency for congruent and incongruent trials (but not the neutral trials) may be a good candidate for the classification task. The response latency among healthy controls is validated to be faster when compared to the patients with schizophrenia across stimulus type. Thus, neutral stimulus type might not be a good candidate for classification task in the feature space. 
TABLE IV

COMPARISON OF BEHAVIORAL PERFORMANCE FOR BOTH SUBJECT CATEGORIES DURING CONGRUENT TRIALS (UPPER PANEL), INCONGRUENT TRIALS (MidDlE Panel), and Neutral TRIals (LOWER Panel) OF THE CMT

\begin{tabular}{|c|c|c|c|}
\hline \multicolumn{2}{|c|}{ Stimulus Type } & \multicolumn{2}{c|}{ Congruent Trials } \\
\hline \multicolumn{2}{|c|}{ Performance Metrics } & Accuracy $(\%)$ & Latency $(\mathrm{ms})$ \\
\hline \multirow{2}{*}{ SZ } & Mean & 71.3 & 1323 \\
\cline { 2 - 4 } & CI & 8.8 & 42.1 \\
\hline \multirow{2}{*}{ HS } & Mean & 73.4 & 1070.2 \\
\cline { 2 - 4 } & CI & 11.2 & 33.4 \\
\hline \multirow{2}{*}{ t-test } & $\mathrm{t}_{(\mathrm{df})}$ Statistic & $\mathrm{t}_{(8)}=-0.35$ & $\mathrm{t}_{(387)}=9.39$ \\
\cline { 2 - 4 } & p-value & 0.73 & $2 \times 10^{-16}$ \\
\hline \multicolumn{2}{|c|}{ Stimulus Type } & \multicolumn{2}{|c|}{ Incongruent Trials } \\
\hline Performance Metrics & Accuracy $(\%)$ & Latency $(\mathrm{ms})$ \\
\hline \multirow{2}{*}{ SZ } & Mean & 65.8 & 1452 \\
\cline { 2 - 4 } & CI & 11.7 & 44.6 \\
\hline \multirow{2}{*}{ HS } & Mean & 70.8 & 1251.2 \\
\cline { 2 - 4 } & CI & 4.7 & 35.8 \\
\hline \multirow{2}{*}{$\mathrm{t}$-test } & $\mathrm{t}_{(\mathrm{df})}$ Statistic & $\mathrm{t}_{(8)}=-0.66$ & $\mathrm{t}_{(370)}=7.0$ \\
\cline { 2 - 4 } & $\mathrm{p}$-value & 0.53 & $1.2 \times 10^{-11}$ \\
\hline
\end{tabular}

\begin{tabular}{|c|c|c|c|}
\hline \multirow{2}{*}{\multicolumn{2}{|c|}{$\begin{array}{c}\text { Stimulus Type } \\
\text { Performance Metrics }\end{array}$}} & \multicolumn{2}{|c|}{ Neutral Trials } \\
\hline & & Accuracy (\%) & Latency (ms) \\
\hline \multirow{2}{*}{ SZ } & Mean & 95.7 & 890.1 \\
\hline & CI & 8.1 & 36.6 \\
\hline \multirow{2}{*}{ HS } & Mean & 98.5 & 862.5 \\
\hline & CI & 0.7 & 20.6 \\
\hline \multirow{2}{*}{ t-test } & $\mathrm{t}_{(\mathrm{df})}$ Statistic & $t_{(8)}=-0.93$ & $\mathrm{t}_{(511)}=1.32$ \\
\hline & $\mathrm{p}$-value & 0.38 & 0.18 \\
\hline
\end{tabular}

CI- 95\% confidence interval; df- degrees of freedom.

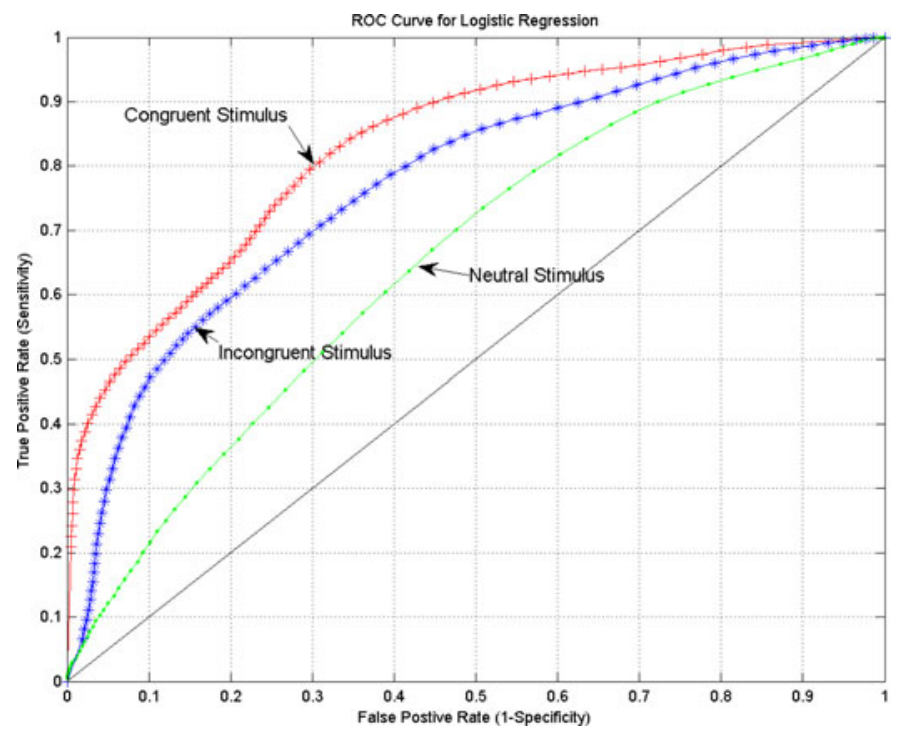

Fig. 2. ROC curve of the classifier for all three stimuli types.

\section{B. Classifier Performance}

The average performance of the classifier for each stimulus type separately trained and tested on 100 randomly selected training and testing sets is reported. The receiver operating characteristics (ROC) curve for the classifier performance is depicted in Fig. 2 for all three stimuli types. The area under the ROC curve measures the performance of the classifier. Higher
TABLE V

Single-Trial Classifier Performance for Each Stimulus Type

\begin{tabular}{|c|c|c|c|}
\hline Stimulus type & Congruent & Incongruent & Neutral \\
\hline $\begin{array}{c}\text { Training set } \\
\text { accuracy \% }\end{array}$ & 74 & 71 & 61 \\
\hline $\begin{array}{c}\text { Testing set } \\
\text { accuracy \% }\end{array}$ & 73 & 69 & 59 \\
\hline $\begin{array}{c}\text { True Positive Rate } \\
\text { (TPR) }\end{array}$ & 0.668 & 0.613 & 0.453 \\
\hline $\begin{array}{c}\text { False Positive } \\
\text { Rate (FPR) }\end{array}$ & 0.212 & 0.23 & 0.29 \\
\hline Area under Curve & 0.83 & 0.76 & 0.64 \\
\hline
\end{tabular}

TABLE VI

Likelihood Ratings Assigned to SubJeCts IN EACH CATEGory ACROSS STIMULUS TYPE

\begin{tabular}{|c|c|c|c|}
\hline $\begin{array}{c}\text { Likelihood } \\
\text { ratings }\end{array}$ & $\begin{array}{c}\text { Duration of } \\
\text { Illness } \\
\text { (months) }\end{array}$ & Congruent & Incongruent \\
\hline \multirow{4}{*}{$\begin{array}{c}\text { Healthy } \\
\text { Control }\end{array}$} & - & 0.24 & 0.25 \\
\cline { 2 - 4 } Subjects & - & 0.36 & 0.51 \\
\cline { 2 - 4 } & - & 0.24 & 0.33 \\
\cline { 2 - 4 } & - & 0.35 & 0.39 \\
\hline \multirow{4}{*}{$\begin{array}{c}\text { Schizophrenia } \\
\text { Subjects }\end{array}$} & - & 0.36 & 0.37 \\
\cline { 2 - 4 } & 120 & 0.62 & 0.64 \\
\cline { 2 - 4 } & 36 & 0.82 & 0.73 \\
\cline { 2 - 4 } & 24 & 0.67 & 0.57 \\
\hline
\end{tabular}

values of the area under the ROC curve indicate a statistically reliable classification performance.

In Table $\mathrm{V}$, we report the average results of 100 training and testing phases for the performance metrics at single trial and at subject level for all three stimuli types. We observe single-trial average classification accuracy of around $73 \%$, and area under the ROC curve of around 0.83 . Thus, we observe that congruent stimulus type performs better than incongruent stimulus type, also confirmed by the ROC curve in Fig. 2. We also note that classification of single-trial using neutral stimulus type leads to $59 \%$ accuracy, which is closer to random guess, confirming our surmise that neutral stimulus type is not favorable for classification using our proposed features.

\section{Likelihood Rating}

The likelihood ratings assigned to all subjects belonging to both healthy and schizophrenia categories for both congruent and incongruent stimulus types are provided in Table VI. We can infer that the likelihood ratings are on the border, i.e., around threshold of 0.5 , for those schizophrenia subjects, in whom the illness has set in very recently as reflected by the duration of illness specified in months.

\section{CONCLUSION}

EEG and MEG are noninvasive tools that acquire brain signals with high temporal resolution using scalp electrodes and provide valuable insights into the systems-level processing associated with rest and cognitive ability. Phase synchronization is one of the most effective signal processing tools to measure task specific ensemble activities of different brain regions specialized 
for specific functions [30], [31]. We proposed the quantity $\gamma$ in (4) to capture a multichannel Hilbert transform-based phase synchronization measure in $0-1$ range, using which it is possible to compare ensembles for level of synchronicity.

If single-trial EEG classification is a challenging problem [13], then task-based classification of EEG of a patient with mental disorder relative to that of a subject without the disorder is even more challenging [14]. Here, we have attempted to develop a single-trial EEG-based classifier that differentiates congruent and incongruent EEG trials in a CMT as belonging to the schizophrenia group or the healthy control group. We have chosen four features for logistic regression from hemispheric ensemble synchronization at three specific intervals in addition to response latency. We believe this approach has good potential for developing a real-time system capable of estimating the likelihood of a subject belonging to the schizophrenia group based on their performance on certain cognitive tasks. One of our future goals will be to validate this method on a large data set, improvising it by including other frequency bands, while limiting to channel clusters relevant to the cognitive task under study.

\section{ACKNOWLEDGMENT}

The authors acknowledge Prof. B. M. Kutty of the National Institute of Mental Health and Neurosciences for inputs regarding experimental design and EEG data acquisition.

\section{REFERENCES}

[1] K. J. Friston, "Functional and effective connectivity in neuroimaging: A synthesis," Hum. Brain Map., vol. 2, pp. 56-78, 1995.

[2] A. Treisman, "A feature integration theory of attention," Cogn. Psychol., vol. 12, no. 1, pp. 97-136, 2003.

[3] S. Palva, K. Linkenkaer-Hansen, R. Naatanen, and J. M. Palva, "Early neural correlates of conscious somatosensory perception," J. Neurosci., vol. 25, no. 21, pp. 5248-5258, 2005.

[4] J. Fell, G. Fernandez, P. Klaver, C. E. Elger, and P. Fries, "Is synchronized gamma activity relevant for selective attention?," Brain Res. Rev., vol. 42, pp. 265-272, 2003.

[5] J. Fell and N. Axmacher, "The role of phase synchronization in memory processes," Nat. Rev. Neurosci., vol. 12, pp. 105-118, 2011.

[6] L. L. Colgin, T. Denninger, M. Fyhn, T. Hafting, T. Bonnevie, O. Jensen, M.-B. Moser, and E. I. Moser, "Frequency of gamma oscillations routes flow of information in the hippocampus," Nature, vol. 462, pp. 353-357, 2009.

[7] N. Axmacher, F. Mormann, G. Fernandez, C. E. Elger, and J. Fell, "Memory formation by neuronal synchronization," Brain Res. Rev., vol. 52, no. 1, pp. 170-182, 2006.

[8] A. K. Engel, P. Fries, and W. Singer, "Dynamic predictions: oscillations and synchrony top-down processing," Nat. Rev. Neurosci., vol. 2, pp. 704716, 2001.

[9] U. Lindenberger, S.-C. Li, W. Gruber, and V. Muller. (2009). Brains swinging in concert: Cortical phase synchronization while playing guitar, $B M C$ Neurosci. [Online]. 10(22). Available: www.biomedcentral.com/1471$2202 / 10 / 22$

[10] P. J. Uhlhaas and W. Singer, "Neural synchrony in brain disorders: Relevance for cognitive dysfunctions and pathophysiology," Neuron, vol. 52, no. 1, pp. 155-168, 2006.

[11] J. Muller-Gerking, G. Pfurtscheller, and H. Flyvbjerg, "Designing optimal spatial filters for single-trial EEG classification in a movement task," Clin. Neurophysiol., vol. 110, no. 5, pp. 787-798, 1999.

[12] E. Haselsteiner and G. Pfurtschelle, "Using time-dependent neural networks for EEG classification," IEEE Trans. Rehabil. Eng., vol. 8, no. 4, pp. 457-463, Dec. 2000.

[13] G. Townsend, B. Graimann, and G. Pfurtscheller, "Continuous EEG classification during motor imagery-simulation of an asynchronous BCI," IEEE Trans. Neural Syst. Rehabil. Eng., vol. 12, no. 2, pp. 258-265, Jun. 2004.
[14] N. Hazarika, J. Z. Chen, A. C. Tsoi, and A. Sergejew, "Classification of EEG signals using the wavelet transform," in Proc. IEEE 13th Int. Conf. Digit. Signal Process. Soc., 1997, pp. 89-92.

[15] N. Kopell, G. B. Ermentrout, M. A. Whittington, and R. D. Traub "Gamma rhythms and beta rhythms have different synchronization properties," Proc. Natl. Acad. Sci. USA, vol. 97, pp. 1867-1872, 2000.

[16] S. L. Bressler, "Cortical coordination dynamics and the disorganization syndrome in schizophrenia," Neuropsychopharmacology, vol. 28 pp. S35-S39, 2003.

[17] K. M. Spencer, P. G. Nestor, M. A. Niznikiewicz, D. F. Salisbury, M. E. Shenton, and R. W. McCarley, "Abnormal neural synchrony in schizophrenia," J. Neurosci., vol. 23, no. 19, pp. 7407-7411, 2003.

[18] ADJUST, an open source EEGLAB plug-in, 2010. [Online]. Available: www.unicog.org/pm/pmwiki.php/MEG/RemovingArtifactsWithADJUST

[19] A. Mognon, J. Jovicich, L. Bruzzone, and M. Buiatti, "ADJUST: An automatic EEG artifact detector based on the joint use of spatial and temporal features," Psychophysiology, vol. 48, no. 2, pp. 229-240, 2011.

[20] A. Delorme and S. Makeig, "EEGLAB: An open source toolbox for analysis of single-trial EEG dynamics including independent component analysis," J. Neurosci. Methods, vol. 134, pp. 9-21, 2004.

[21] M. Rosenblum, A. Pikovsky, J. Kurths, C. Schafer, and P. A. Tass, "Phase synchronization: From theory to data analysis," in Neuro-Informatics and Neural Modeling, F. Moss and S. Gielen, Eds. Amsterdam, The Netherlands: Elsevier, 2001, pp. 279-321.

[22] P. D. Prasad, S. Vishaka Datta, and K. Majumdar, "Enhanced phase and amplitude synchronization towards focal seizure offset," Clin. EEG Neurosci., vol. 44, no. 1, pp. 16-24, 2013.

[23] J. Gallinat, G. Winterer, C. S. Herrmann, and D. Senkowski, "Reduced oscillatory gamma-band responses in unmedicated schizophrenic patients indicate impaired frontal network processing," Clin. Neurophysiol., vol. 115 , pp. 1863-1874, 2004.

[24] A. R. Haig, E. Gordon, V. de Pascalis, R. A. Meares, H. Bahramali, and A. Harris, "Gamma activity in schizophrenia: Evidence of impaired network binding?" Clin. Neurophysiol., vol. 111, pp. 1461-1468, 2000.

[25] E. Gordon, L. Williams, A. R. Haig, J. Wright, and R. A. Mear, "Symptom profile and 'gamma' processing in schizophrenia," Cog. Neuropsychiatry, vol. 6, no. 1, pp. 7-19, 2001.

[26] J. S. Kwon, B. F. O’Donnell, G. V. Wallenstein, R. W. Greene, Y. Hirayasu, P. G. Nestor, M. E. Hasselmo, G. F. Potts, M. E. Shenton, and R. W. McCarley, "Gamma frequency-range abnormalities to auditory stimulation in schizophrenia," Arch. Gen. Psychiatry, vol. 56, no. 11, pp. 1001-1005, 1999.

[27] P. Tass, M. G.. Rosenblum, J. Weule, J. Kurths, A. Pikovsky, J. Volkmann, A. Schnitzler, and H-J. Freund, "Detection of $\mathrm{n}$ : $\mathrm{m}$ phase locking noisy data: Application to magnetoencephalography," Phys. Rev. Lett., vol. 81 no. 15 , pp. 3291-3294, 1998.

[28] R. Bhatia, Matrix Analysis. New York, USA: Springer-Verlag, 1997.

[29] J.-P. Lachaux, A. Lutz, D. Raudrauf, D. Cosmelli, M. L. Van Quyen, J. Martinerie, and F. Varela, "Estimating the time-course of coherence between single-trial brain signals: An introduction to wavelet coherence," Clin. Neurophsiol., vol. 32, no. 3, pp. 157-174, 2002.

[30] D. Rudrauf, A. Douiri, C. Kovach, J.-P. Lachaux, D. Cosmelli, M. Chavez, C. Adam, B. Renault, J. Martinerie, and M. L. Van Quyen, "Frequency flows and the time-frequency dynamics of multivariate phase synchronization in brain signals," NeuroImage, vol. 31, no. 1, pp. 209-227, 2006.

[31] C. Allefeld and J. Kurths, "An approach to multivariate phase synchronization analysis and its application to event-related potentials," Int. J. Bifurc. Chaos, vol. 14, no. 2, pp. 417-426, 2004.

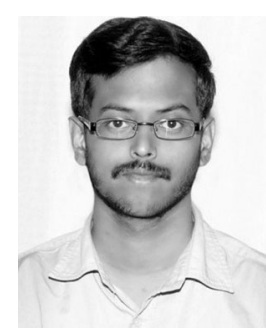

Pradeep D. Prasad (M'03) received the B.Tech. degree in electronics and communication engineering from the National Institute of Technology Tiruchirappalli, Trichy, Tamil Nadu, India, in 2006.

He has worked in various positions in the industry for five years in the field of signal processing and its applications. Till recently, he was with the Systems Science and Informatics Unit, Indian Statistical Institute, Bangalore Center, India. His research interests include signal processing and its applications to neuroscience. 


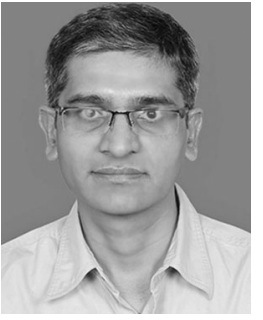

Harsha N. Halahalli received the M.B.B.S. degree from Karnatak Medical College, Karnatak University, Karnatak, India, in 1997, the MD degree in physiology from the Jawaharlal Institute of Post-Graduate Medical Education and Research, Pondicherry University, Puducherry, India, in 2001, and the M.Phil. and Ph.D. degrees in neurophysiology from the National Institute of Mental Health and Neuro Sciences (NIMHANS), Bangalore, India, in 2004 and 2013, respectively.

He was a Research Associate at the Multimodal Brain Image Analysis Laboratory, NIMHANS and a Faculty Member at K. S. Hegde Medical Academy, Nitte University, Mangalore, India, where he currently a Professor of physiology.

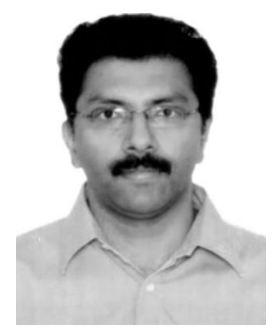

John P. John received the M.B.B.S. degree from Mahatma Gandhi University, Kerala, India, in 1994, and the M.D. degree in Psychiatry from the National Institute of Mental Health and Neurosciences (NIMHANS), Bangalore, India, in 1999. He underwent postdoctoral training in MRI analysis at the Washington University School of Medicine, St. Louis, MO, USA, in 2005.

Since 2002, he has been a Faculty Member in the Department of Psychiatry, NIMHANS, Bangalore, India. He currently holds the position of Additional Professor of Psychiatry as well as an Adjunct Faculty in the Department of Clinical Neuroscience. He is also the Officer-in-Charge of the Multimodal Brain Image Analysis Laboratory at the NIMHANS.

Dr. John's awards and honors include the Fogarty International ICOHRTA fellowship in 2003, Young Investigator Award for the best paper at the Second International Congress of Biological Psychiatry in 2007, the Young Psychiatrist Award in the Asian Region instituted by the Royal Perth Hospital in 2008, and the Tilak Venkoba Rao Oration Award of the Indian Psychiatric Society in 2008.

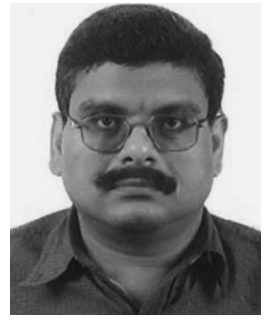

Kaushik K. Majumdar (M'06-SM'10) received the B.Sc. degree from the University of Calcutta, Kolkata, India, in 1990, the M.Sc. degree in mathematics from the Annamalai University, Annamalai Nagar, India, 1996, and the M.Tech. and Ph.D. degrees in computer science from the Indian Statistical Institute, Kolkata, India, in 1999 and 2003, respectively.

He was with the University of Memphis, the University of Oregon, and Florida Atlantic University, all in USA. He was also with INRIA Sophia Antipolis in France. He is currently an Associate Professor at the Indian Statistical Institute, Bangalore Center, India, where he heads the Computational Neuroscience Group. His interests include neural signal processing (EEG, ECoG, LFP, and fMRI), mathematical and computational modeling of the brain functions, and nonlinear system theoretic studies of epilepsy.

Dr. Majumdar is a member of the American Mathematical Society and the Society for Neuroscience. 